\title{
Application of Sensitivity Encoding Reconstruction for MRI with BOLD Signal
}

\author{
Lianjun Zhang, Gang Liu \\ Computer College Science and Technology Shandong University of Technology, Zibo, China \\ Email: zhlj0512@163.com
}

How to cite this paper: Zhang, L.J. and Liu, G. (2021) Application of Sensitivity Encoding Reconstruction for MRI with BOLD Signal. Journal of Computer and Communications, 9, 27-34.

https://doi.org/10.4236/jcc.2021.94002

Received: March 26, 2021

Accepted: April 13, 2021

Published: April 16, 2021

Copyright $\odot 2021$ by author(s) and Scientific Research Publishing Inc. This work is licensed under the Creative Commons Attribution International License (CC BY 4.0).

http://creativecommons.org/licenses/by/4.0/

\begin{abstract}
The work of this paper analyzes the performance of Sensitivity Encoding (SENSE) through actual data sets and determines the problem of computational efficiency. It corrects the error of the detection signal through the calibration function of the percentage signal change, and uses the three-dimensional sensor image reconstruction technology to calibrate the sensitivity of the blood to the magnetic change, enhances the sensitivity of the magnetic susceptibility gradient, and reduces the scanning time of the MRI experiment. The actual data set handles the image resolution. The performance and experimental results of SENSE are analyzed through actual data sets.
\end{abstract}

\section{Keywords}

BOLD Signal, Sensitivity Encoding, MRI, Reconstruction

\section{Introduction}

The magnetic resonance imaging (MRI) to detect blood oxygenation level dependent (BOLD) signal is the most common tool. It can be done by detecting the magnetic susceptibility of blood, which depends on the oxygenation state of hemoglobin. Based on cognitive neuroscience non-invasive examination of the brain's participation function, due to the increase in oxygen consumption and blood flow caused by the activity of brain neurons, the amount of oxygenated hemoglobin will increase, and the change in the amount of oxygenated hemoglobin will cause paramagnetic effects, magnetic resonance Imaging can detect this $T_{2}^{*}$-weighted signal.

The MRI detects the magnetic susceptibility of blood which is sensitive to changes in the microscopic magnetic susceptibility. MRI images the difference in the macroscopic magnetic susceptibility from the interface between the air and 
the tissue. The difference in magnetic susceptibility between air and tissue will seriously damage the uniformity of the magnetic field in the surrounding area. The non-uniformity caused by the magnetic susceptibility can cause loss of image signal. Many development and optimization methods have been developed to correct these artifacts from the inhomogeneity of the magnetic susceptibility field [1] [2] [3] [4].

The magnetic field gradient affects the BOLD sensitivity of MRI acquisition. The magnetic field gradient generated by the inhomogeneity of the macroscopic magnetic field will destroy the spatial frequency sensitivity code of blood. Among these schemes, the most famous may be Sensitivity Encoding (SENSE) [5] and SMASH [6]. SENSE has its own advantages in providing parallel imaging with arbitrary coil configurations, albeit at the expense of SNR. Both of these techniques can significantly compress the scan time, but usually a computationally intensive inverse process is needed to expand the image from insufficient sampled data, especially in the case of non-Cartesian data sets. These calculation problems need to be improved and the algorithm popularized. The reconstructed three-dimensional MRI data set can be used to identify certain areas of the brain that have significant differences in age-related functional sensitivity. Calibrate the BOLD sensitivity change caused by the magnetic field change, and outline the area of significant sensitivity change to solve.

The intensity of blood magnetization depends on the oxygenation state of hemoglobin, and changes in the oxygenation state of hemoglobin will affect the magnetic susceptibility of blood. Due to the increase in oxygen consumption and blood flow caused by neuronal activity, the amount of oxidized hemoglobin in this area will increase, which will lead to paramagnetic effects and changes in MRI signals. The magnetic susceptibility gradient can change the change of BOLD sensitivity. Sensitivity coding (SENSE) enhances the detection of magnetic susceptibility gradient sensitivity, reduces the scan time of MRI experiments, and maintains image resolution through under-sampling data sets.

\section{Blood Oxygenation Level Dependent Sensitivity Encoding in Magnetic Resonance Imaging}

The MRI imaging of BOLD sensitivity coding is usually achieved by placing a receiving coil array around the object to be imaged to detect changes in magnetic susceptibility. Independent receiving coils provide spatially different received signals to the collected data set. In a typical MRI experiment, the formation of the baseband image for the following equation shows:

$$
\rho(t)=\int d(r) S(r) \mathrm{e}^{-i \omega(r) t} \mathrm{e}^{-i 2 \pi k(t) r} \mathrm{~d} r+\varepsilon(t),
$$

The $d(r)$ is the continuous function of the transverse magnetization of the object at position $r$, its trajectory can be directly obtained from the frequency space $\mathrm{k}$ space; $S(r)$ is the spatial sensitivity curve of the receiver coil; $\omega(r)$ is the $B_{0} \mathrm{Un}$ iformity; $k(t)$ is the input data trajectory of the independent variable at time $t$; $\varepsilon(t)$ is the noise term at time $t$. Equation (1) shows that k-space data, coil profile 
sensitivity and magnetic field inhomogeneity information are the three basic elements of parallel imaging. Each sensitivity received by the coil is its own data $S(r) . \rho_{1}$ represents the signal vector received from the coil 1 , and the image vector $\rho$ is formed by stacking into a single column. $S$ is the diagonal matrix, the spatial sensitivity distribution maintained in 1 coils; $F$ is the imaging system matrix.

Sensitivity encoding imaging uses signal processing methods to reduce the scan time and the amount of MRI data obtained, while still maintaining the same spatial resolution. From the perspective of phase, the data obtained by the array coil is correct. However, according to the sampling theory, the imaging of the sensitivity coding scheme should reduce the number of phase coding lines at an interval of $1 /$ field of view (FOV). When using conventional FFT reconstruction, the alias will be displayed in the reconstructed image.

The SENSE imaging scheme [7] combines the above-mentioned aliasing effects and aliasing signals from multiple coils to achieve faster imaging speed and reduce the error between aliasing. Taking single-point sampling MRI as an example, the position and distance between the phase code lines should be known before the MRI experiment. Based on these positions and distances, the number of pseudonyms in the reconstructed image of the coil can be accurately calculated. If the scanning time is shortened by the $\mathrm{R}$ acceleration factor, the FOV in the reconstructed image will be reduced or collapsed by the same $R$ factor. The signal intensity obtained by reconstructing the aliased pixel enhances the signal intensity at the desired position, and the signal intensity is superimposed with integer multiples of $F O V / R$ to replace the original pixel value. The SENSE scheme can superimpose the image intensity and filter out the expected error value.

The working mode of the SENSE imaging scheme is: To obtain the required image from the acquired complete FOV image data, the image value of the pixel $(x, y)$ should be in the corresponding position $\left(x, y_{l}\right)$, with the coil sensitivity profile $S$ Weighted, where $l=1, \cdots, R$. The accurate reconstructed pixel value of the position $(x, y)$ with $k^{\prime}$ coil information is follows:

$$
I_{k}(x, y)=S_{k}\left(x, y_{1}\right) \rho\left(x, y_{1}\right)+S_{k}\left(x, y_{2}\right) \rho\left(x, y_{2}\right)+\cdots+S_{k}\left(x, y_{R}\right) \rho\left(x, y_{R}\right)
$$

This equation is converted to matrix notation and illustrated in Figure 1 below:
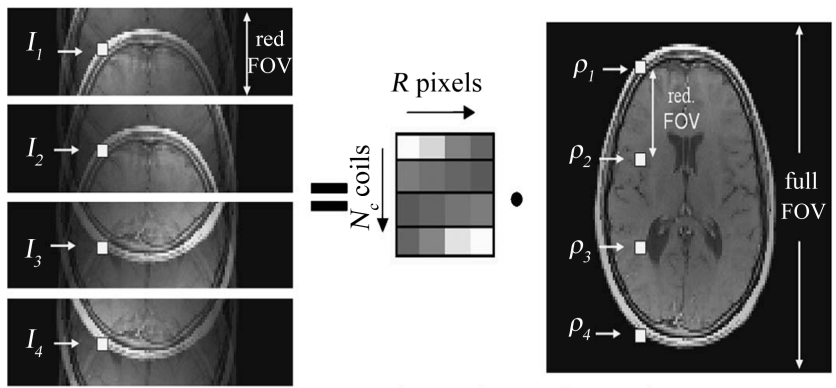

Figure 1. The SENSE imaging scheme. 


$$
\boldsymbol{I}=\boldsymbol{S} \cdot \boldsymbol{\rho}
$$

In order to simplify the formula as Equation (4), can do not consider the noise correlation problem. According to the matrix theory knowledge, by summarizing the sensitivity matrix $S$, easily obtain a complete FOV image, the $S$ :

$$
\begin{gathered}
\boldsymbol{\rho}=\left(\boldsymbol{S}^{H} \boldsymbol{S}\right)^{-1} \boldsymbol{S}^{H} \cdot \boldsymbol{I} \\
\rho(\boldsymbol{r})=\int_{-\infty}^{\infty} S(\boldsymbol{k}) \mathrm{e}^{i 2 \pi \boldsymbol{k} \cdot \boldsymbol{r}} \mathrm{d} \boldsymbol{k}
\end{gathered}
$$

The characteristics of the SENS process in this equation are realized by general matrix inversion. It is worth noting that the SENSE reconstruction algorithm needs to be executed at each pixel position in the folded $F O V$ image to finally reconstruct the complete FOV image. In the actual implementation of SENS reconstruction, due to memory problems and computational bottlenecks, we usually do not apply the explicitly formed matrices $S$ and $S^{H}$ to general matrix inversion solutions, and usually use the combined gradient method to find the solution results in an iterative manner.

The technique for creating internal images, using a persistent area of activity is magnetic resonance imaging. MRI can detect signal changes caused by brain nerve activity. Deoxygenated hemoglobin is quasi-magnetic, while fully oxidized hemoglobin is diagnosable and more similar to surrounding tissues. In the process of neural activity, Deoxygenated hemoglobin will make the static magnetic field more distorted and the MRI signal will be attenuated. The different frequencies predicted in the non-uniform magnetic field will lead to the problem of rapid phase cancellation. Changes in blood oxidation will cause changes in the MRI attenuation parameter $T_{2}^{*}$ and change the $T_{2}^{*}$-weighted image. The blood oxidation level depends on the detection of MRI, which is usually achieved by taking a series of snapshots every two seconds and minutes. Check how the measured brain signals are related to the brain stimulation area presented, and the relative changes in the concentration of deoxygenated hemoglobin.

Due to the difference in susceptibility between air and tissue, changes in the magnetic field exist in the brain area near the air/tissue interface. The magnetic space generated by the imaging gradient is not susceptible

$$
K_{\text {imaging }}(t)=\gamma \int_{0}^{t} G_{\text {imaging }}(\tau) \mathrm{d} \tau
$$

After calculating the susceptibility gradient, the position of the k-space depends on the susceptibility gradient. The generated with the susceptibility gradient is

$$
K_{\text {susc }}(t)=\gamma \int_{0}^{t} G_{\text {susc }}(\tau) \mathrm{d} \tau
$$

Since $G_{\text {susc }}$ is a constant ( $\left.G_{\text {susc }}(t)=G_{\text {susc }} \forall t\right)$, Simplify the above formula to

$$
K_{\text {susc }}(t)=\gamma G_{\text {susc }} t
$$

The calculated magnetic susceptibility gradient is

$$
K(t)=K_{\text {imaging }}(t)+K_{\text {susc }}(t)=\gamma \int_{0}^{t} G_{\text {imaging }}(\tau) \mathrm{d} \tau+\gamma G_{\text {susc }}\left(t+t_{0}\right)
$$


The data collection starts from $t_{0}$ with the value of the $R F$ pulse and the data collection duration affecting the spatial trajectory will tilt. If the susceptibility gradient is positive, in the same direction as the gradient without susceptibility, the starting point of the spatial trajectory will move downward due to the susceptibility gradient between the $R F$ pulse and the data acquisition. Due to the influence of the magnetic susceptibility gradient, the trajectory will tilt downwards, it will pass the origin quickly, and the echo time will be shorter than originally planned; if the magnetic susceptibility gradient is negative, the same derivation also applies. This is because the gradient is reversed and there is no magnetic susceptibility. The starting point of the space orbit will move upward, the echo time $t$ will be longer.

\section{Experimental Results and Discussion}

As tissues with the same susceptibility, the magnetic field gradients be no induced by susceptibility, too there will be no echo times. And the echo time of the signal will be the same. In reality, the magnetic field will produce a gradient due to changes in the magnetic susceptibility near the air/tissue interface. The magnetic susceptibility gradient will be displayed on the k-space trajectory, and the echo time is measured at the origin. If the k-space trajectory is shifted and skewed, the echo time may be different than expected. MRI signal of BOLD echo time is used to optimize signal contrast. If the echo time changes, it will affect the BOLD signal strength and affect the MRI display.

Without the susceptibility gradient transformation, PSC is defined as:

$$
\operatorname{PSC}\left(T E_{\text {nom }}\right)=100 * \frac{S_{\text {active }}-S_{\text {rest }}}{S_{\text {rest }}}=100 * \frac{S_{0}\left(\mathrm{e}^{-\frac{T E_{\text {nom }}}{T_{2, \text { active }}^{*}}}-\mathrm{e}^{-\frac{T E_{\text {nom }}}{T_{2, \text { rest }}}}\right)}{S_{0} \mathrm{e}^{-\frac{T E_{\text {nom }}}{T_{2, \text { rest }}^{*}}}}
$$

$S_{\text {active }}$ is the MRI signal received when the organization is in the active state, $S_{\text {rest }}$ is the received signal, $S_{0}$ is the voxel image intensity, $T E_{\text {nom }}$ is echo time, $T_{2, \text { rest }}^{*}$ is the $T_{2}^{*}$ relaxation time resting state, $T_{2, \text { active }}^{*}$ is the $T_{2}^{*}$ relaxation time constant during active state. $T_{2, \text { active }}^{*}=49.6 \mathrm{~ms}, T E_{\text {nom }}=30.0 \mathrm{~ms}$, and $T_{2, \text { rest }}^{*}=48.9 \mathrm{~ms}$ [8]. If add susceptibility gradient, the space will move and the echo time will also change. PSC with a susceptibility gradient can be defined:

$$
\operatorname{PSC}\left(T E_{\text {eff }}\right)=100 * \frac{S_{0}\left(\mathrm{e}^{-\frac{T E_{\text {eff }}}{T_{2, \text { cative }}^{*}}}-\mathrm{e}^{-\frac{T E_{\text {eff }}}{T_{2, \text { est }}^{*}}}\right)}{S_{0} \mathrm{e}^{-\frac{T E_{\text {eff }}}{T_{2, \text { est }}^{*}}}}
$$

Use the information in the measured magnetic field to predict how the echo time will change and estimate the BOLD signal that will be generated. A calibration function is formed to eliminate the expected change BOLD signal due to the magnetic susceptibility gradient. The calibration functions are as follows: 


$$
\operatorname{Calibration}\left(T E_{\text {eff }}\right)=\frac{P S C\left(T E_{\text {eff }}\right)}{P S C\left(T E_{\text {nom }}\right)}
$$

Comparing the old and young people from different data sets from previous functional imaging studies, test results [9] [10] [11]. The experimental subject group included thirty young adults (18 - 21 years old, average age 18.8, 18 women) [12]. The Siemens (Erlangen, Germany) Allegra 3T MRI scanner was used to scan the subjects. Use the following parameters: $T E=10.00,12.46 \mathrm{~ms}$, $T R=700 \mathrm{~ms}$, field of view $=22 \mathrm{~cm}$, basic resolution $=64$, phase resolution $=72$, bandwidth $=260$. For the young group, use the same protocol to obtain the magnetic field map, the difference is: $T R=1000 \mathrm{~ms}, 38$ slices. For the unit of the field image, the gradient of the field image can be found by calculating the difference with the adjacent pixels in the field image, and the unit of the gradient is $\mathrm{Hz} / \mathrm{cm}$.

To find $T E_{\text {eff }}$, to find $K_{\text {eff }}$ which is the sum of $K_{\text {imaging }}$ and $K_{\text {susc }}$. By the definition of $P S C\left(T E_{\text {eff }}\right)$ a calibration function in each voxel can be calculated. There is a significant difference between $T E_{\text {eff }}$ and the calibration $\left(T E_{\text {eff }}\right)$ of the data of the young group and the old group. Figure 2 are bar graphs showing the means areas of significant difference. Each ROI and its $\log _{10}$ ( $p$-values) the standard deviation of the two groups, as well as images of the brain, some of the significant areas have large age differences (blue The colored area represents the area where the $\mathrm{p}$ value is between $10^{-2}$ to $10^{-5}$ ).

The magnetic field caused by cerebral blood oxygen saturation sensitivity can lead to changes in the sensitivity of functional imaging of different brain regions. If there is a net deviation between the two groups of people who are comparing brain function, the net deviation from the sensitivity can be shown as a functional change across ages. The interaction between measurement techniques and other potential net changes between groups must be understood to ensure that functional differences are correctly explained [13] [14].

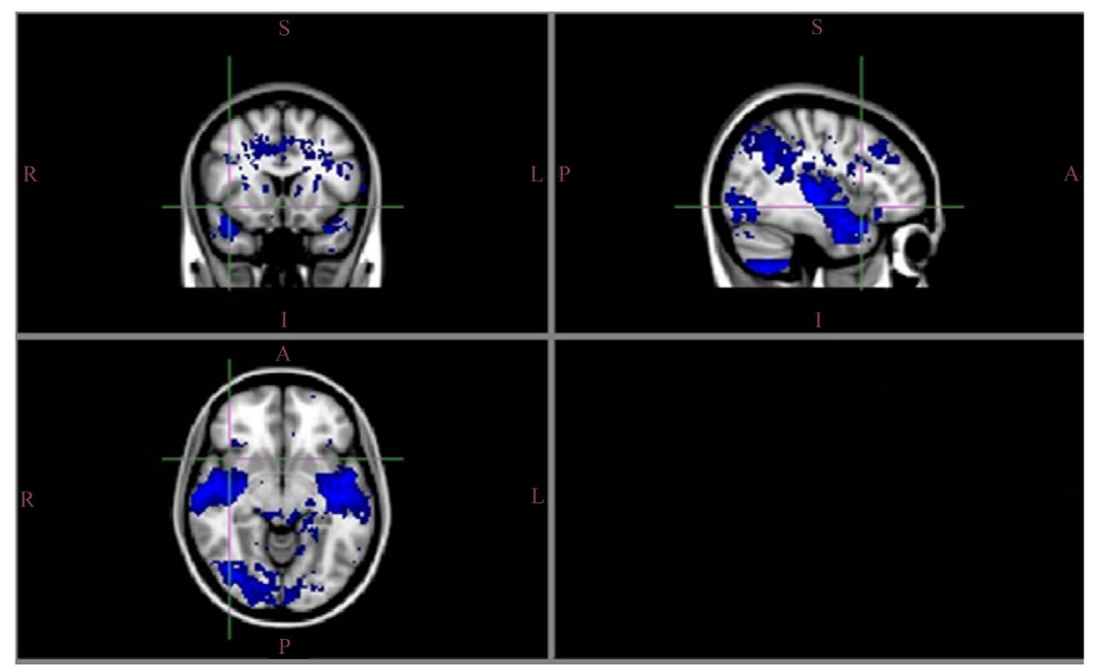

Figure 2. The Areas of significant difference Map of $\log _{10}(p s c)$. 
Applying three-dimensional SENSE to the image reconstruction technology I am studying, calibration may help eliminate the sensitivity deviation between the two groups. By measuring a single specific magnetic field distribution and calculating the expected BOLD sensitivity, a more obvious improvement can be obtained.

\section{Conclusion}

The blood oxygenation level dependent signal (BOLD) is used for functional magnetic resonance imaging (MRI), which uses the blood magnetization to depend on the oxygenation state of hemoglobin. The magnetic susceptibility gradient shift of the blood magnetic field will cause the MRI echo time shift and the BOLD sensitivity change. Use the calibration function of percentage signal change to correct the error of the detection signal, and use the three-dimensional SENSE image reconstruction technology to calibrate the sensitivity of BOLD to magnetic changes. The reconstructed image contains a small amount of Gaussian white noise, which can be eliminated by SENSE to improve the resolution of magnetic resonance imaging. The performance and experimental results of this method are calculated and analyzed through actual data. This method can be applied to many different fields, such as the research of age-related blood oxygenation level-dependent signal detection, and the problem of further improving the calculation speed is analyzed.

\section{Conflicts of Interest}

The authors declare no conflicts of interest regarding the publication of this paper.

\section{References}

[1] Deichmann, R., Josephs, O., Hutton, C., Corfield, D.R. and Turner, R. (2002) Compensation of Susceptibility-Induced BOLD Sensitivity Losses in Echo-Planar fMRI Imaging. Neuroimage, 15, 120-135. https://doi.org/10.1006/nimg.2001.0985

[2] Truong, T., Clymer, B.D., Chakeres, D.W. and Schmalbrock, P. (2002) Three- Dimensional Numerical Simulations of Susceptibility-Induced Magnetic Field Inhomogeneities in the Human Head. Magnetic Resonance Imaging, 20, 759-770. https://doi.org/10.1016/S0730-725X(02)00601-X

[3] Reber, P.J., Wong, E.C., Buxton, R.B. and Frank, L.R. (1998) Correction of off Resonance-Related Distortion in Echo-Planar Imaging Using EPI-Based Field Maps. Magnetic Resonance in Medicine, 39, 328-330.

https://doi.org/10.1002/mrm.1910390223

[4] Sutton, B.P., Noll, D.C. and Fessler, J.A. (2003) Fast, Iterative Image Reconstruction for MRI in the Presence of Field Inhomogeneities. IEEE Transactions on Medical Imaging, 22, 178-188. https://doi.org/10.1109/TMI.2002.808360

[5] Pruessmann, K. (1999) SENSE: Sensitivity Encoding for Fast MRI. Magnetic Resonance in Medicine, 42, 952-962.

https://doi.org/10.1002/(SICI)1522-2594(199911)42:5<952::AID-MRM16>3.0.CO;2$\underline{\mathrm{S}}$

[6] Pruessmann, K. (2001) Advances in Sensitivity Encoding with Arbitary k-Space 
Trajectories. Magnetic Resonance in Medicine, 46, 638-651. https://doi.org/10.1002/mrm.1241

[7] Fessler, J. (2005) Toeplitz-Based Iterative Image Reconstruction for MRI with Correction for Magnetic Field Inhomogeneity. IEEE Transactions on Signal Processing, 53, 3393-3402. https://doi.org/10.1109/TSP.2005.853152

[8] Woodard, J. and Sugarman, M. (2011) Functional Magnetic Resonance Imaging in Aging and Dementia: Detection of Age-Related Cognitive Changes and Prediction of Cognitive Decline. Current Topics in Behavioral Neurosciences, 10, 113-136. https://doi.org/10.1007/7854_2011_159

[9] Zambri, B. and Djellouli, R. (2017) An Efficient Multistage Algorithm for Full Calibration of the Hemodynamic Model from BOLD Signal Responses. International Journal for Numerical Methods in Biomedical Engineering, 33, 2875-2879. https://doi.org/10.1002/cnm.2875

[10] Erickson, K.I., Voss, M.W., Prakash, R.S., Basak, C., Szabo, A., Chaddock, L., Kim, J.S., Heo, S., Alves, H., White, S.M., Wojcicki, T.R., Mailey, E., Vieira, V.J., Martin, S.A., Pence, B.D., Woods, J.A., McAuley, E. and Kramer, A.F. (2011) Exercise training Increases Size of Hippocampus and Improves Memory. Proceedings of the National Academy of Sciences, 108, 3017-3022.

https://doi.org/10.1073/pnas.1015950108

[11] Voss, M.W., Prakash, R.S., Erickson, K.I., Boot, W.R., Basak, C., Neider, M.B., Simons, D.J., Fabiani, M., Gratton, G. and Kramer, A.F. (2012) Effects of Training Strategies Implemented in a Complex Videogame on Functional Connectivity of Attentional Networks. Neuroimage, 59, 138-148. https://doi.org/10.1016/j.neuroimage.2011.03.052

[12] Warren, S.L. (2010) Effects of Adult Attachment and Emotional Distractors on Brain Mechanisms of Cognitive Control. Psychological Science, 21, 1818-1822. https://doi.org/10.1177/0956797610388809

[13] Spielberg, J.M., Miller, G.A., Engels, A.S., Herrington, J.D., Sutton, B.P., Banich, M.T. and Heller, W. (2011) Trait Approach and Avoidance Motivation Lateralized Neural Activity Associated with Executive Function. Neuroimage, 54, 661-670. https://doi.org/10.1016/j.neuroimage.2010.08.037

[14] Matsubara, T., Torikai, H.S. and Leibnitz, K. (2014) A Nonlinear Model of FMRI BOLD Signal Including the Trend Component. Proceedings of the International Joint Conference on Neural Networks, 4 September 2014, Beijing, 2579-2586. https://doi.org/10.1109/IJCNN.2014.6889646 